decision receiving specialist palliative care in hospital, hospice or community settings.

Results 73 HCP's participated in the survey and 87\% had discussions about CPR. In HCPs having conversations about CPR $75 \%$ rated their confidence in doing so as $8 / 10$ or higher.

187 case notes were reviewed. While DNACPR forms indicated whether the decision had been discussed with the patient in $87 \%$ only $72 \%$ had a record of this in the written notes. $68 \%$ of case notes had a record of discussions or reasons for non-discussion with those important to the patient.

$34 \%$ of patients moved care setting after the DNACPR decision, of these $64 \%$ transferred with a unified DNACPR form.

Conclusion This audit shows ongoing challenges in communicating with patients and those important to them about CPR. Improvement is required in disseminating DNACPR decisions when patients transfer care settings.

\section{TRANSFER OF CARE FROM HOSPITAL TO HOME IN THE LAST DAYS OF LIFE: IS IT SAFE AND EFFECTIVE?}

Adam Hurlow, Rose Laud, Arpit Patel, Deborah Borrill. Leeds Teaching Hospitals NHS Trust, University of Leeds School of Medicine, University of Leeds School of Medicine, Leeds Teaching Hospitals NHS Trust

\subsection{6/bmjspcare-2018-ASPabstracts.80}

Background Hospital teams must plan a safe and effective transfer of care for patients returning home in the last days of life. To support this Leeds Teaching Hospitals NHS Trust (LTHT) launched a revised Rapid Discharge Plan (RDP) in January 2014.

Aim To assess the quality of transfer of care (ToC) for patients returning home from LTHT who died within a week of discharge (June 2014 - May 2015).

Methods A convenience sample of 45 patients was selected from a database of 228. Clinical notes were reviewed for recognition of dying (prognosis days) pre-discharge, RDP use, and evidence of eight key interventions necessary for a successful ToC.

Results Median survival from discharge was 4 (1-7) days. Thirty-one (69\%) had a progressive life-limiting illness and 12 (27\%) had multi-morbidity/frailty.

Twenty-five patients (56\%) were recognised to be dying. Key interventions took place for the majority, including: advance care planning (ACP) (96\%), Fast Track discharge (92\%), anticipatory prescribing $(88 \%)$ and do not attempt cardiopulmonary resuscitation form (DNACPR) (84\%).

The RDP was used in 11 (44\%) of those recognised to be dying. The RDP patients had a median of six (5-8) key interventions compared to four (1-5) for those without an RDP.

Eight $(18 \%)$ were perceived to be in the last weeks to months of life and twelve (27\%) were not recognised to be approaching the EoL at all. Ten (83\%) of those not recognised to be near the EoL had multi-morbidity/frailty, whereas $29(87 \%)$ of patients in the other two groups had a life-limiting illness. The majority $(73 \%)$ of those not recognised had two or more markers of deteriorating health.

Conclusion Appropriate planning occurred for the majority of patients recognised to be dying. This was enhanced by use of the RDP. Recognition is a barrier to planning; particularly in those with multi-morbidity and frailty.
54 ARE MODERN DAY PALLIATIVE CARE NURSES RITUAL SPECIALISTS?

Lindsey Buster, Laura Green, Christina Faull, Karina Croucher. University of Bradford, LOROS

\subsection{6/bmjspcare-2018-ASPabstracts.81}

Background Recent research in palliative care has focused on understanding how the dying (and immediately post-mortem) process affects nurses. The literature notes the increasingly complex environments in which nurses find themselves, such as in the care of heart-beating and non-heart-beating cadaver donors, whose legal status as variously 'alive' or 'dead' is often less clear-cut in practice. Nurses often report feelings of emotional 'burnout' due to a lack of suitable support structures.

Methods A review of literature from 1980 to the present, predominantly from specialist nursing journals was consulted to provide an overview on the role and emotional responses of contemporary palliative care nurses. The findings were contextualised using research frameworks derived from common themes in archaeological and anthropological literature, such as personhood and liminality.

Results Themes such as the liminality of the dead are recurrent in both modern palliative nursing and in the archaeological and ethnographic record. Moreover, the need for ritual specialists to mediate in transitions between the worlds of the living of the dead is ubiquitous in the latter, as is the elevated status of these individuals within the community.

Conclusions By understanding the important and conflicted nature of their roles as mediators between worlds, and by placing their daily practices within a broader theoretical framework and deep-time perspective, nurses can better understand the inherently complex and sensitive nature of their work, and gain a degree of empowerment over their roles and associated emotional responses.

\section{THE UTILITY OF A TRUSTWIDE CONSULTATION TO PROMOTE AND INFORM THE IMPLEMENTATION OF AN END OF LIFE CARE STRATEGY}

Emma Shereston, Emma Dove, Paul Whiting, Eleanor Smith. Sheffield Teaching Hospitals NHS Foundation Trust

\subsection{6/bmjspcare-2018-ASPabstracts.82}

Background The Trust's Strategy for End of Life Care (EOLC) outlines a vision that ensures care at end of life is as comfortable, dignified and individualised as possible. The importance of staff engagement to achieve success and effective implementation of the Strategy was recognised and the following outlines how this was approached.

Method A stakeholder analysis was undertaken to identify target staff groups and invitations were sent through a variety of communication methods. Consultation events were held with staff at all levels (clinical and non-clinical) during March and April 2017, led by Service Improvement, across a variety of Trust sites. These took the form of short interactive sessions lasting $30 \mathrm{~min}$ : a short presentation explaining the project background, followed by the opportunity to comment on 3 specific areas - what works well/could be improved for patients; for staff; and what would help improve staff confidence in delivering EOLC.

Results 140 staff attended from a wide range of professions; 891 comments were received, collated, analysed and grouped 